\title{
Oral Pyruvate Effects on Dorsal Root Ganglia in Simulated Weightlessness Rats
}

\author{
Yuan Li ${ }^{\mathrm{a}}$, Heng Zhang ${ }^{\mathrm{b}}$, Ning Tao Ren ${ }^{\mathrm{c}}$, Chao Chen ${ }^{\mathrm{a}}$, Peng Qi ${ }^{\mathrm{b}}$, \\ Fang Qiang Zhou ${ }^{\mathrm{d}, \mathrm{e}}$, Geng Cui ${ }^{\mathrm{a}}$, e
}

\begin{abstract}
Background: Long-term microgravity in spaceflight has been demonstrated with induction of multi-organ damage and dysfunction, including the dorsal root ganglia (DRG) injury. Present studies investigated protective effects of oral pyruvate in drinking water on lumbar 5 (L5) DRG tissues in rats subjected with hindlimb unweighting (HU).

Methods: Sprague-Dawley male rats were randomly divided into four groups $(\mathrm{N}=10)$ : the control (CON) group, the suspension (SUS) group, the normal saline (SAL) group and the sodium pyruvate (PYR) group, respectively. Rats in the SUS, SAL and PYR groups were treated with simulated microgravity by $\mathrm{HU}$ with tail suspension for an 8-week period. Rats in the SAL and PYR groups were fed with normal saline and pyruvate saline, respectively. Histopathological hematoxylin-eosin (H\&E) staining and Nissl-stained neurons as well as myelin sheath immunofluorescence examinations in L5 DRG tissues were carried out. The protein expression of glial cell line-derived neurotrophic factor (GDNF), glial fibrillary acidic protein (GFAP), neuron-specific enolase (NSE) and S100 calcium-binding protein B (S100B) as well as the levels of adenosine triphosphate (ATP) and adenosine triphosphatase (ATPase) were also determined after HU procedures.
\end{abstract}

Results: Data showed that nerve tissues' structure and function were robustly altered in L5 DRG of the SUS and SAL groups, whereas morphological changes were not significantly different between the PYR group and the CON group; levels of GDNF, GFAP, NSE and S100B were significantly changed in the SUS and SAL groups, but

Manuscript submitted October 15, 2021, accepted February 23, 2022

Published online February 28, 2022

anstitute of Orthopedics, the First Medical Center, Chinese PLA General Hospital, Beijing 100853, China

${ }^{b}$ Department of Emergency, the First Medical Center, Chinese PLA General Hospital, Beijing 100853, China

'Department of Orthopedics, the Fourth Medical Center of Chinese PLA General Hospital, Beijing 100037, China

dShanghai Sandai Pharmaceutical R\&D Company, Ltd, Shanghai 201203, China

${ }^{\mathrm{e}}$ Corresponding Author: Fang Qiang Zhou, Shanghai Sandai Pharmaceutical R\&D Company, Ltd, Shanghai 201203, China. Email: fqzh60130@yahoo. com; Geng Cui, Department of Orthopedics, Chinese PLA General Hospital, Beijing 100853, China. Email: cuigeng@aliyun.com

doi: https://doi.org/10.14740/jnr709 mostly preserved or overexpressed in group PYR following the HU injury. However, levels of ATP and ATPase were significantly retained, but still worse in the PYR group than in the CON group. The significance of oral pyruvate protection against DRG injury following the $\mathrm{HU}$ and the dose and formula of oral pyruvate solutions were discussed for the use of astronauts in space missions.

Conclusions: This study indicates that oral pyruvate can effectively protect L5 DRG against the damage of pathological alterations and dysfunction induced by the $\mathrm{HU}$ in rats. Further investigations are needed.

Keywords: Dorsal root ganglia; Glial cell line-derived neurotrophic factor; Hindlimb unweighting; Neuron; Pyruvate

\section{Introduction}

Many previous studies demonstrate that astronauts are subjected to physiological and metabolic alterations and multi-organ injury in spaceflights, including bone loss and muscle atrophy, cerebral and spinal cord injury (SCI), cardiovascular, immune system disorders and so on. However, effective interventions are still lacking [1-5].

Dorsal root ganglia (DRG) as a critical anatomical structure play a crucial role in the transmission of sensation of pain injury. Many neurotrophic factors and neurotransmitters are also synthetized in the DRG, which exert important impacts on the nervous nutrition and injury repair. The changes of neurotrophic factor expressions and genomics and the degenerative alteration of myelin sheath occur in the fifth lumbar spine (L5) DRG in simulated microgravity, as shown previously in rats $[4,5]$. These aberrances illustrate the microscopic nervous damage.

Pyruvate is an important intermediate product of glycolysis, which connects glucose anaerobic and aerobic metabolism. It is not only an energy substrate, but also an anti-inflammatory and anti-oxidative stress agent. Pyruvate possesses several beneficial physiological and pharmacological properties in the prevention from glucometabolic disturbances and the protection against mitochondrial dysfunction. Therefore, it is multiorgan protective against various pathogenic stimuli [6-8]. Several studies have demonstrated that pyruvate has apparently protective effects against central and peripheral nerve damage 
induced by various noxious insults [9-11]. Importantly, recent findings demonstrated that a novel pyruvate-enriched oral rehydration solution ((Pyr-ORS: equimolar pyruvate substitute of citrate or bicarbonate in the World Health Organizationdirected ORS (WHO-ORS)) robustly preserved visceral blood flow and organ function, corrected hypoxic lactic acidosis and enhanced survival in oral rehydration of animals subjected to severe shock [11-13]. Enteral pyruvate in Pyr-ORS as intravenous pyruvate also remarkably sustained cerebral neurons' structure and function following ashyxial cardiac arrest in animal studies $[11,14]$.

Following our prior findings [5], the present study focuses on investigation of protective effects of oral pyruvate on the DRG injury in rats subjected to simulated microgravity, further highlighting the changes in the histopathological structure and metabolism of DRG nervous cells, and displays the first evidence for oral pyruvate in the prevention and treatment of the back pain and beyond for astronauts in spaceflight missions.

\section{Materials and Methods}

\section{Animal and tissue preparation}

Forty 3-month-old Sprague-Dawley male rats $(180 \pm 20 \mathrm{~g})$, obtained from the Animal Experimental Center of the Academy of Military Medical Sciences, Beijing, were assigned to four groups $(\mathrm{N}=10)$ : the control $(\mathrm{CON})$ group, the suspension (SUS) group, the normal saline (SAL) group and the sodium pyruvate saline (PYR) group. The rats in SUS, SAL and PYR groups were simulated with microgravity effects in the hindlimb unweighting (HU) model by the tail suspension [8]. The hind limbs (legs) of rats were held off the horizontal plane approximately at a $30^{\circ}$ angle. Rats in groups CON and SUS were fed with regular laboratory water. The rats in group SAL and PYR were orally taken with $0.9 \%$ sodium chloride solution (normal saline, NS, $\left[\mathrm{Na}^{+}\right] 154 \mathrm{mmol} / \mathrm{L},\left[\mathrm{Cl}^{-}\right] 154 \mathrm{mmol} / \mathrm{L}$ ) and sodium pyruvate saline $\left(\left[\mathrm{Na}^{+}\right] 154 \mathrm{mmol} / \mathrm{L},\left[\mathrm{Cl}^{-}\right] 104 \mathrm{mmol} / \mathrm{L}\right.$ and $\left[\mathrm{Pyr}^{-}\right] 50 \mathrm{mmol} / \mathrm{L}$ ) daily to replace the conventional laboratory water during the experiment, respectively. Pyruvate saline was prepared in the laboratory following Zhou's invention patent formula in $2006: 0.61 \% \mathrm{NaCl}+0.55 \% \mathrm{NaPyr}$. The total volume of drinking water fed daily was about $30 \pm 10 \mathrm{~mL}$ each rat. Experimental rats were subjected with $\mathrm{HU}$ for a period of 8 weeks following 1 week of adaptive suspension. Rats were placed in a 12-h light/dark cycle with the standard laboratory light and food during experimentation. Rats were anesthetized by sodium pentobarbital (intraperitoneally (IP) $40 \mathrm{mg} / \mathrm{kg}$ ), sacrificed and dissected after the 8-week experiment. The bilateral lumbar 5 (L5) DRG of rats, which were in the size of about $1-1.5 \mathrm{~mm}$ each ganglion, were quickly excised and frozen in liquid nitrogen with isopentane except the part of tissues for regular hematoxylin-eosin (H\&E) staining [5]. Sodium pyruvate was obtained from Sigma Co. (St. Louis, MO) and normal saline (Cisen Pharmaceutical Co., China) was acquired from the Animal Laboratory of PLA General Hospital, Beijing.

In each rat, part of the tissue was used for histopathological examinations and the rest was for other determinations from each dorsal root ganglion ( $\mathrm{n}=20$, bilateral $\mathrm{DRG})$. A cryotome cryostat (Microm, Heidelberg, Germany) was used for cryopreservation of the frozen tissue blocks prior to sectioning and other determinations.

The protocols in the experiment were approved by the Guidelines of Animal Testing Committee in the PLA General Hospital, Beijing, China. Animal care and treatment procedures were according to the Guiding Principles for Animal Care and Use of the Department of Physiology and Sciences, Beijing, China.

\section{H\&E staining}

A part of the L5 DRG tissue sample in each group was fixed in formalin for $24 \mathrm{~h}$, washed and dehydrated by ethanol gradient, as described previously [5]. The tissue blocks were regularly embedded in paraffin treated with dimethyl benzene and then sliced in cross-sections. The tissue sections were incubated at $60{ }^{\circ} \mathrm{C}$ for $1 \mathrm{~h}$, finally dewaxed with xylene, dehydrated with ethanol and conventionally stained with H\&E. A light microscope was used for the H\&E stain examination.

\section{Nissl body with toluidine blue staining}

Briefly, one section was selected in every five-section interval, tissue sections of bilateral DRG $(n=20)$ were washed by phosphate buffered saline (PBS) placed on the coverslips and then fixed in $4 \%$ paraformaldehyde for $30 \mathrm{~min}$ and stained with $1 \%$ toluidine blue at room temperature for $30 \mathrm{~min}$. The tissue sections were then washed with absolute ethanol, dried and finally coverslipped [5]. The sections were analyzed with a light microscopy $(\times 400$, OLYMPUS BX46). The researchers blindly observed the experimental samples. The quantities of Nissl-stained neurons per $1 \mathrm{~mm}^{2}$ region of the five random areas were counted each slice in average. Finally, totally five slices with well staining were randomly selected from bilateral DRG and averagely evaluated in each rat $(\mathrm{N}=10)$, using the optical microscopy with the image tool software (Nikon NISElements, USA).

\section{Myelin sheath immunofluorescence}

A series of continuous cross-section of bilateral L5 DRG tissues $(n=20)$ were conducted at a $20-\mu \mathrm{m}$ thick and one section was selected in every five-section interval for the immunofluorescence determination to evaluate the expressions of myelin sheath, as described previously [15]. Briefly, for staining, the tissue sections were thawed at room temperature for $30 \mathrm{~min}$. Tissue sections were washed with PBS and incubated with 3\% hydrogen peroxide for $10 \mathrm{~min}$. Then, sections were rinsed by normal goat serum at room temperature for $2 \mathrm{~h}$ and incubated with the primary antibody of polyclonal degenerate myelin basic protein (dgen-MBP) (1:1,000, Chemicon Inc., Temecula, USA) at $4{ }^{\circ} \mathrm{C}$ overnight. After rinsing again, tissue sections were further incubated at $4{ }^{\circ} \mathrm{C}$ overnight with the secondary 
antibody of donkey anti-rabbit IgG-FITC (1:200, Jackson ImmunoResearch Laboratories Inc., West Grove, PA, USA). Next, sections were washed three times with PBS for $10 \mathrm{~min}$ each time. At last, tissue sections were mounted, using Floursave mounting reagent (Cal Biochem, La Jolla, USA). Finally, five slices with optimal staining were randomly chosen per rat for the evaluation $(\mathrm{N}=10)$. The fluorescence intensity of images was collected and comparisons among groups were carried out following the stained area and the mean integrated optical density (OD). The mean OD values were analyzed as a semi-quantitative measurement index, using Image $\mathrm{J}$ software (National institute of Health, USA).

\section{Western blot analysis}

The L5 DRG tissues were prepared for conducting Western blotting, as previously described $[5,16]$. Briefly, bilateral DRG proteins from part of the tissues in each rat $(\mathrm{N}=10)$ were extracted by the experimental standard method and the protein contents were determined by the Bradford method. The cell lysates were separated, using SDS-PAGE, then transferred to the PVDF membranes. The membranes were blocked in TBST accommodated $5 \%$ skim milk at room temperature for $30 \mathrm{~min}$, followed by incubation with a primary antibody $(1: 1,000$, rabbit anti-rat glial cell-derived neurotrophic factor (GDNF) (ab64337, Abcam); 1:1,000, rabbit anti-rat glial fibrillary acidic protein (GFAP) (ab138701, Abcam); 1:4,000, rabbit anti-rat neuron-specific enolase (NSE) (ab180943, Abcam); 1:500, rabbit anti-rat S100 calcium-binding protein $\mathrm{B}(\mathrm{S} 100 \mathrm{~B})$ (ab80508, Abcam) and 1:5,000, anti- $\beta$-actin (ab95437, Abcam)) at $4{ }^{\circ} \mathrm{C}$ overnight, respectively. The blots were washed by TBST and incubated in a secondary antibody (goat anti-rabbit IgG $\left(1: 2,000\right.$, Abcam)) at $37{ }^{\circ} \mathrm{C}$ for $1 \mathrm{~h}$. The protein expressions were visualized with the enhanced chemiluminescence (ECL) reagent (Amersham, Cleveland, $\mathrm{OH}$, USA) and each membrane was exposed to Hyperfilm (Amersham). The intensity values of GDNF, GFAP, NSE and S100B were measured, using the Quantity-One software (BIO-RAD, USA) in comparison to $\beta$-chain. The relative ratios were expressed with results for the four proteins, respectively.

\section{Tissue ATP content}

A part of the bilateral L5 DRG tissues in each rat $(n=20)$ was quickly homogenized for preparing the tissue lysate, then, protein concentrations were determined. The ATP level in DRG tissues $(\mathrm{N}=10)$ was measured by absorbance values, as described previously [17]. Tissue ATP contents were determined stringently according to the instructions obtained with the test kit (model: A095-1-1) acquired from Nanjing Jiancheng Bioengineering Institute, China. At last, the absorbance value at $636 \mathrm{~nm}$ was read with each tube by high-performance liquid chromatography (HPLC, Agilent Co., USA). The ATP contents of test samples were calculated, according to the following formula provided by the test kit: ATP content $(\mathrm{nmol} / \mathrm{gprot})=($ test tube-control tube $) /($ standard tube-blank tube $) \times$ standard content $\times$ dilution times $\div$ protein $($ gprot $/ \mathrm{L})$

\section{Tissue ATPase activity}

Strictly according to the ATPase test kit (model: NKATP-1-Y) procedures (SuZhou Comin Biotechnology Co., Ltd, China), part of bilateral L5 DRG tissues $(n=20)$ was accurately weighed; the weight over volume ratio of 1:9 was prepared by adding $0.9 \%$ sodium chloride solution to make the enzyme-enriched samples $(\mathrm{N}=10)$. The sample was homogenized, then, centrifuged at $2,000 \mathrm{rpm}$ at $4{ }^{\circ} \mathrm{C}$ for $10 \mathrm{~min}$. The $0.2 \mathrm{~mL}$ of supernatant was taken and diluted with $0.8 \mathrm{~mL}$ of $0.9 \%$ sodium chloride to make a fresh homogenate placed over ice for next determinations. Then, enzymatic and phosphorylation reactions were performed following the operation table in the test kit. At last, the amount of inorganic phosphoric acid was calculated by colorimetric determination at $660 \mathrm{~nm}$ (Agilent Co., USA). ATPase activities were calculated according to the following formula provided by the test kit: ATPase activity ( $\mu \mathrm{mol} / \mathrm{mgprot} / \mathrm{h}$ ) $=($ test tube-control tube $) /($ standard tube-blank tube $) \times$ standard content $\times$ dilution times $\times 7.5 \div$ protein $(\mathrm{mgprot} / \mathrm{mL})$

\section{Data analysis}

The SPSS 17.0 software was employed for the statistical analysis of data expressed in mean \pm standard deviation (SD). Oneway analyses of variance (ANOVA) were performed to explore the significant differences in means among four groups. In addition, the Student's $t$-test was carried out to perform the pairwise comparisons for the differences between each two groups. Statistically significant difference was determined as $\mathrm{P}<0.05$.

\section{Results}

\section{Pyruvate effects on tissue morphology in L5 DRG}

\section{$H \&$ E staining}

The DRG nervous cells displayed slightly swelling and a few of cytoplasm vacuoles in group PYR, compared to those in the CON group. On contrast, the DRG cells appeared quite swelling, gap widened in various levels, some cytoplasmic vacuoles and an abundance of fine granular basophilic materials among cells in groups SUS and SAL. These alterations showed more obvious in groups SUS and SAL than in groups CON and PYR. The cellular morphological appearance in the group PYR was just mildly changed and close to that in the CON group (Fig. 1).

\section{Nissl body of neuron}

Nissl basophilic granules were abundant as near normal appearances in neurons in groups CON and PYR. However, morphological sizes and distribution patterns of Nissl bodies were smaller and scattered, which were also stained lightly, in groups SUS and SAL. The Nissl bodies in some DRG neurons also appeared disassociated each other in these two groups. Further, the quantified 

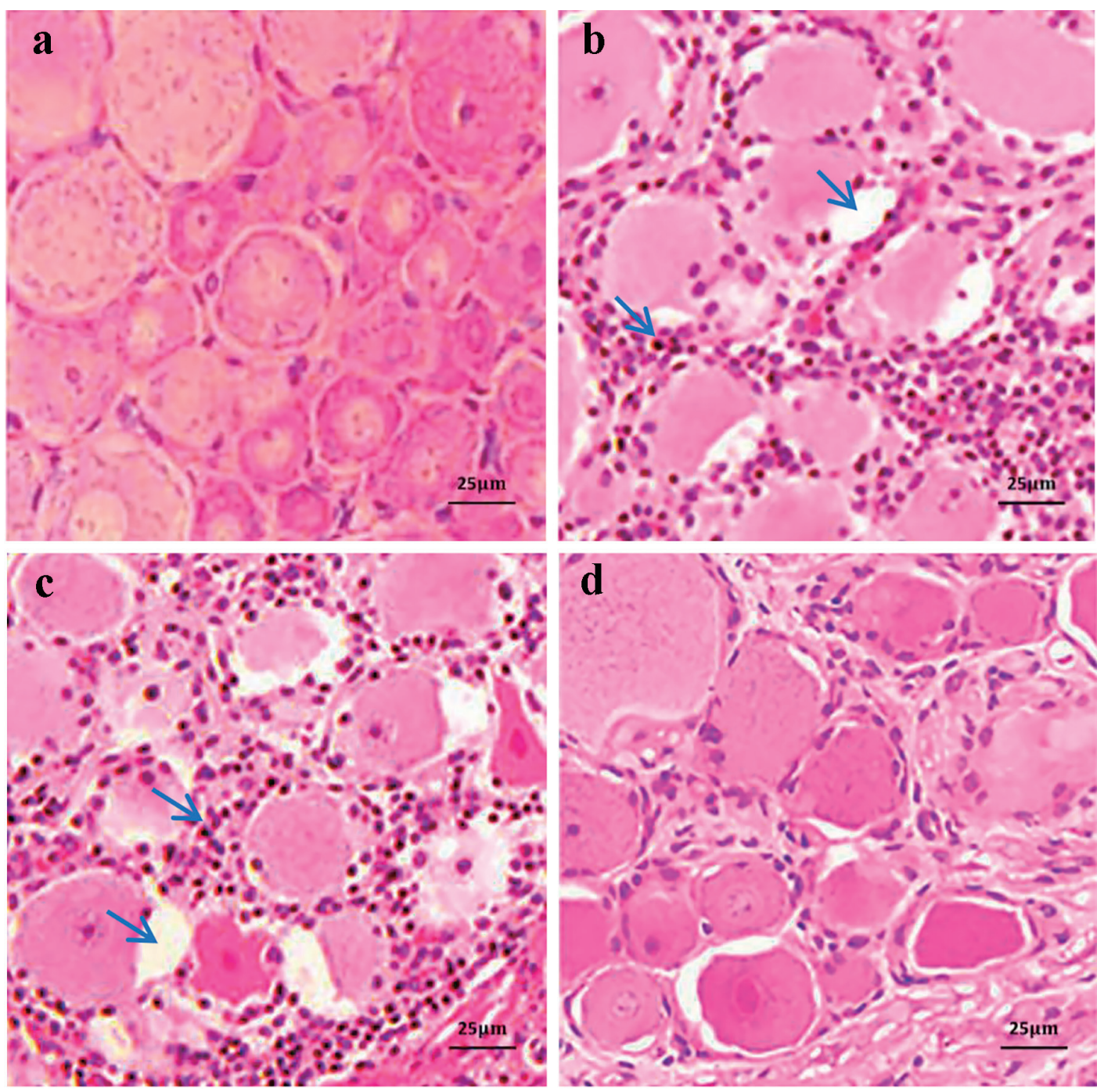

Figure 1. Hematoxylin-eosin (H\&E) staining of L5 dorsal root ganglia (DRG) tissues in various treatment groups. In the sodium pyruvate (PYR) group, the DRG neurons showed only mild swelling and a small amount of cytoplasm vacuoles. In the suspension (SUS) and normal saline (SAL) groups, the neurons presented quite swelling, gap widened in varying degrees and some cytoplasmic vacuoles and there were numerous fine granular basophilic materials among the cells, as shown by arrows at cytoplasmic vacuoles and basophilic granules, respectively. The cell morphological appearance in group PYR was slightly changed in comparison with that in control (CON) group (scale bars $25 \mu \mathrm{m}$ ). (a) Group CON. (b) Group SUS. (c) Group SAL. (d) Group PYR.

alterations in neuron numbers with normal stained Nissl body were significantly lower in groups SUS and SAL than in groups $\mathrm{CON}$ and PYR. The numbers of stained Nissl body neurons had no difference between the two latter groups (Fig. 2A, B).

\section{Degenerated-MBP expression}

In groups SUS and SAL, cell numbers with dgen-MBP were increased relative to groups CON and PYR; the cells were inflated and cellular boundaries were vague with dense fluorescent intensity in the immunofluorescence determination. These changes were not significantly shown in groups CON and PYR (Fig. 3A). The mean OD values of dgen-MBP, which reflected the fluorescence intensity, were significantly enhanced in the SUS and SAL groups, relative to the CON and PYR groups. There were statistically significant differences in mean OD values between the SUS and SAL groups from the CON and PYR groups, as shown in Figure 3B $(\mathrm{P}<0.01)$. However, no difference between both latter groups was found in the mean $\mathrm{OD}$ value $(\mathrm{P}>0.05)$.

\section{Pyruvate effects on GDNF, GFAP, NSE and S100B protein expressions in L5 DRG}

\section{GDNF expression}

As shown in Figure 4, compared to group CON, simulated microgravity for 8 weeks resulted in a robust reduction in relative 

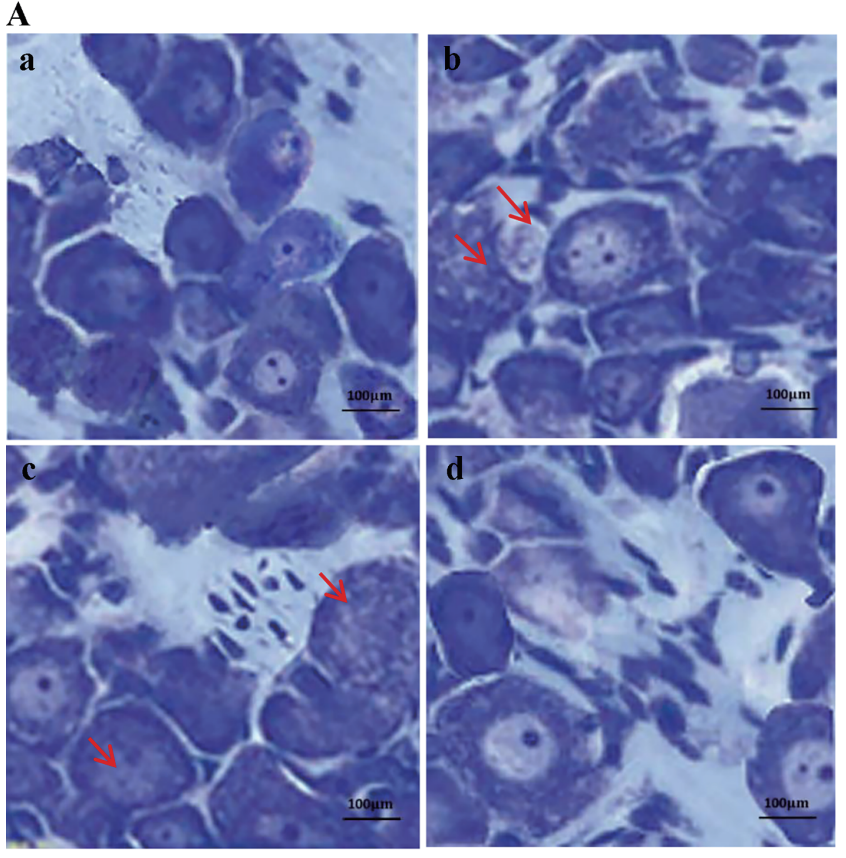

B

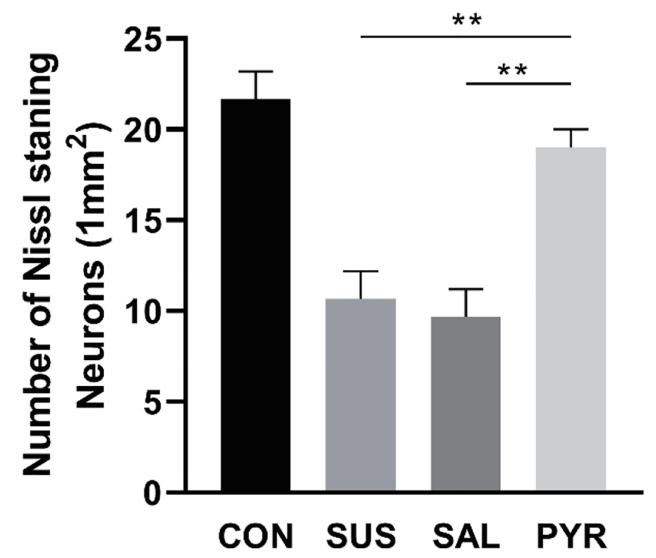

Figure 2. (A) Toluidine blue staining of Nissl bodies in L5 dorsal root ganglia (DRG) neurons in various treatment groups. In the control (CON) and sodium pyruvate (PYR) groups, Nissl basophilic granules were abundant with near normal appearance. However, in the suspension (SUS) and normal saline (SAL) groups, the Nissl bodies were lightly stained with smaller morphological sizes and scattered distribution. In some DRG neurons, the Nissl bodies also appeared disassociated in groups SUS and SAL, as shown by arrows (scale bars $100 \mu \mathrm{m}$ ). (a) Group CON. (b) Group SUS. (c) Group SAL. (d) Group PYR. (B) Quantified analysis of Nissl body staining in various treatment groups. The amounts of Nisslstained neurons $/ 1.0 \mathrm{~mm}^{2}$ in five random fields were semi-quantified by using a light microscope $(\times 400$; five section slices were evaluated in average from bilateral DRG in each rat; $N=10$ per group). The observed and quantified changes were more obvious in the SUS and SAL groups than in the CON and PYR groups and there was no significant difference in numbers of Nissl body staining neurons between groups $\mathrm{CON}$ and PYR. There were statistically significant differences between groups SUS and SAL from group PYR, respectively $\left({ }^{* *} \mathrm{P}<0.01\right)$.

expressions of GDNF proteins in groups SUS and SAL by $21 \%$ and $20 \%$, respectively. On contrast, the GDNF expressions in group PYR were $123 \%$ and $112 \%$ higher than those in groups
A
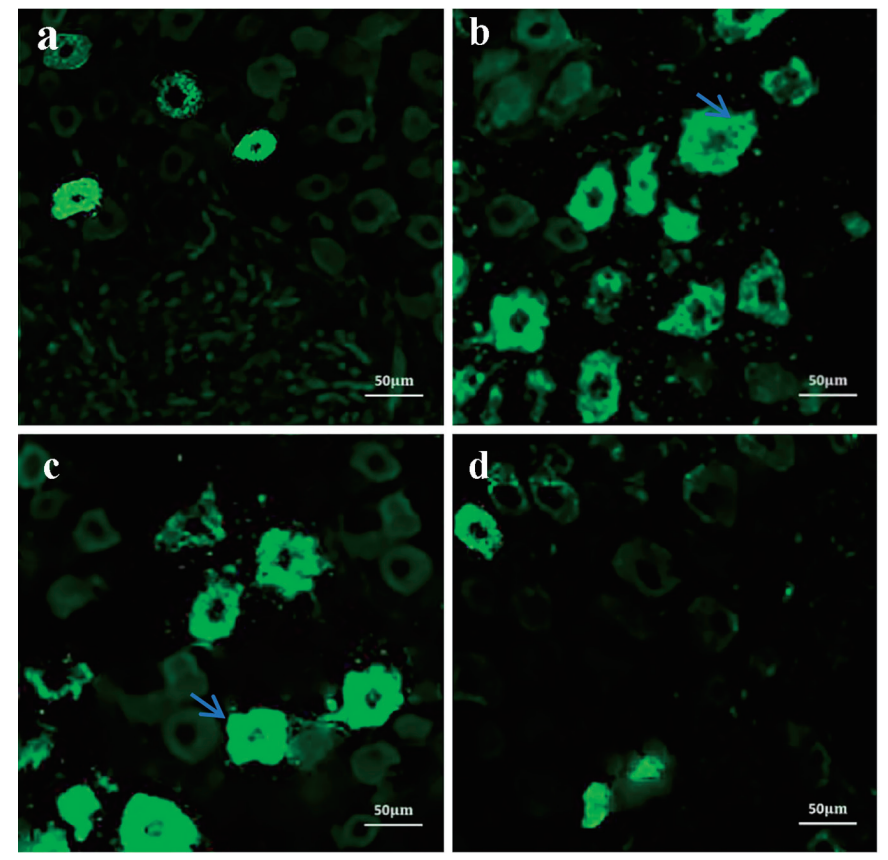

B

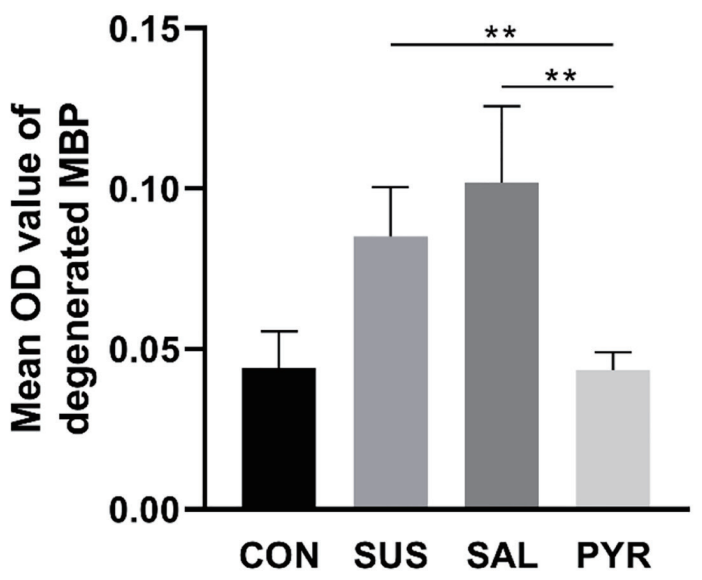

Figure 3. (A) Immunofluorescence detection of L5 dorsal root ganglia (DRG) degenerated myelin basic protein (dgen-MBP) in various treatment groups. The suspension (SUS) and normal saline (SAL) groups showed more expressions of immunofluorescent intensity of dgen-MBP than those in the control (CON) and sodium pyruvate (PYR) groups, as shown by arrows (scale bars $50 \mu \mathrm{m}$ ). (a) Group CON. (b) Group SUS. (c) Group SAL. (d) Group PYR. (B) Mean optical density (OD) value of dgen-MBP in various treatment groups. The immunofluorescent intensity of dgen-MBP was semi-quantitively determined in average from five slices with optimal staining randomly chosen from bilateral DRG per rat ( $\mathrm{N}=10$ per group). Mean OD values of dgen-MBP immunofluorescence staining in L5 DRG were higher in groups SUS and SAL than in groups CON and PYR. There were statistically significant differences between groups SUS and SAL from group PYR, respectively ( $\left.{ }^{* *} P<0.01\right)$.

SUS and SAL, respectively. Statistically significant differences were shown in comparison of group PYR to groups SUA, $\mathrm{SAL}$ and $\mathrm{CON}$, respectively $(\mathrm{P}<0.01)$. Notably, the content of 

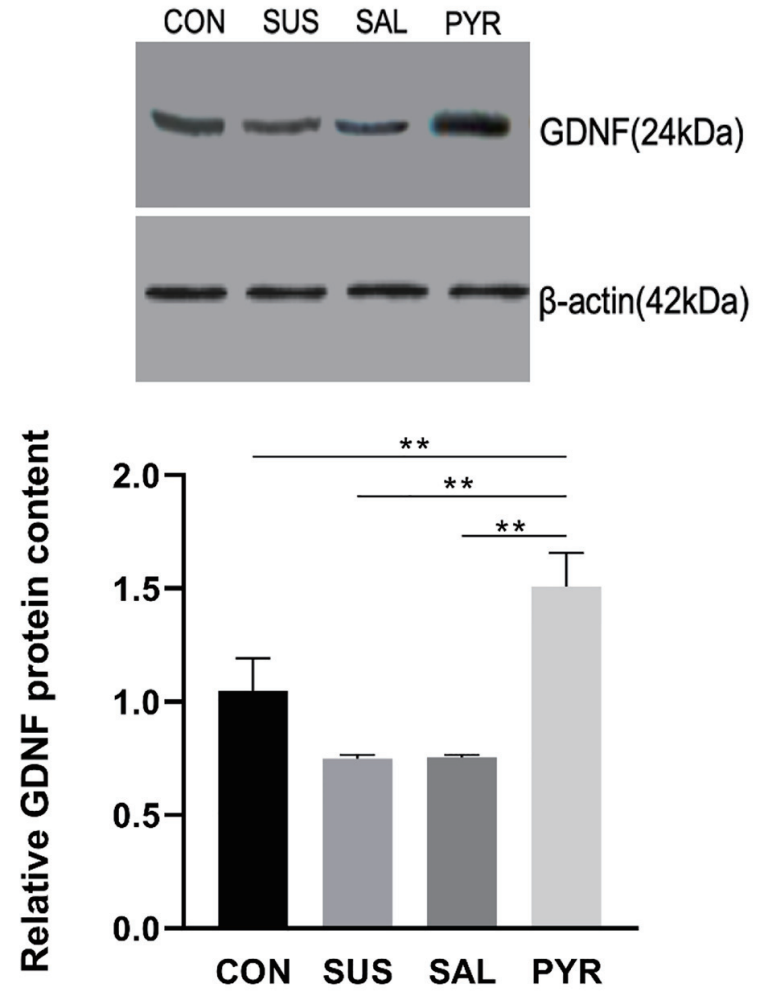

Figure 4. Relative glial cell line-derived neurotrophic factor (GDNF) expressions by Western blotting in L5 dorsal root ganglia (DRG) neurons in various treatment groups. Simulated microgravity induced significant reduction in GDNF expressions. There were statistically significant differences between the suspension (SUS) and normal saline (SAL) groups from the control $(C O N)$ and sodium pyruvate $(P Y R)$ groups $(n=20$ per group). However, the protein content was significantly higher in group PYR than in group CON. There were statistically significant differences between groups CON, SUS and SAL from group PYR, respectively ( $\left.{ }^{* *} \mathrm{P}<0.01\right)$.

GDNF protein was more abundant in the PYR group than in the CON group $(\mathrm{P}<0.01)$.

\section{GFAP expression}

As shown in Figure 5, the relative GFAP protein expressions were significantly augmented in three suspension groups, relative to the CON group. The GFAP contents were around 2.5 times and 2.3 times in the SUS and SAL groups over the CON group, respectively. But the GFAP protein in the PYR group was decreased by $28 \%$ and $24 \%$, relative to the SUS and SAL groups, respectively. There was a statistically significant difference in the GFAP expression between the SUA and SAL groups from the CON and PYR groups $(\mathrm{P}<0.01)$. Also, the content of GFAP expression was still significantly richer in the PYR group than in the CON group $(\mathrm{P}<0.01)$.

\section{NSE expression}

The relative NES protein expression was decreased in the SUS
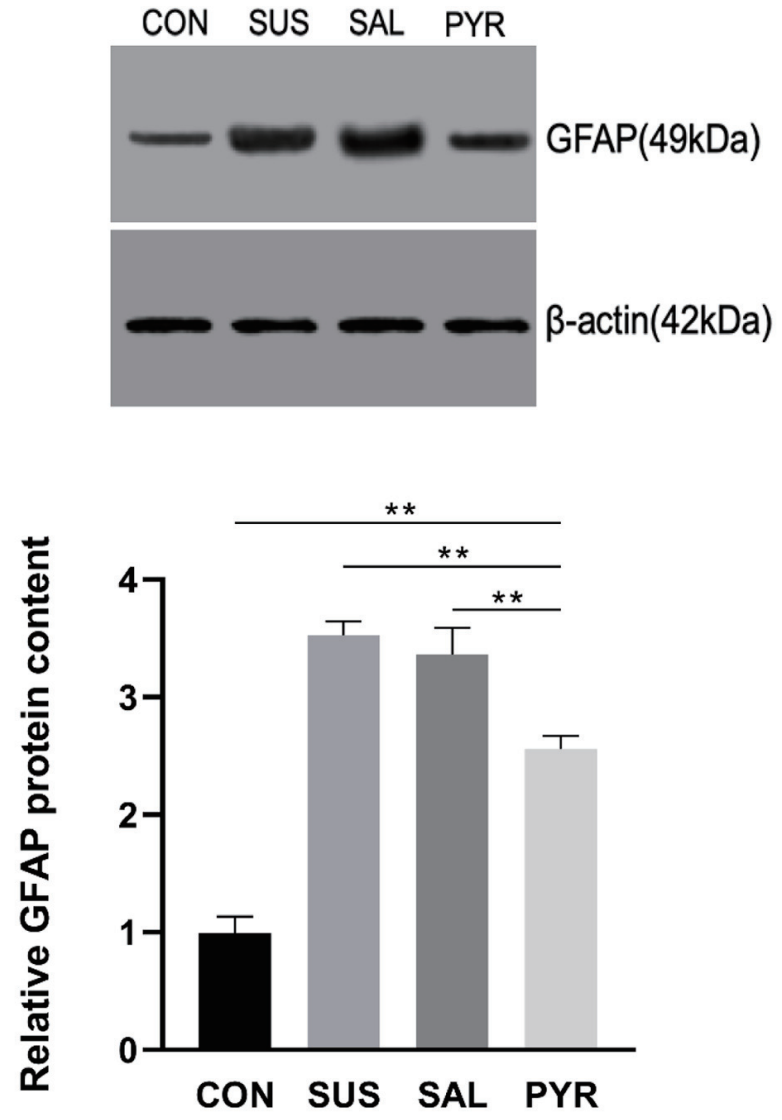

Figure 5. Relative glial fibrillary acidic protein (GFAP) expressions by Western blotting in L5 dorsal root ganglia (DRG) tissues in various treatment groups. The GFAP protein expressions were significantly increased in all three suspension groups. There were statistically significant differences between groups suspension (SUS) and normal saline (SAL) from groups control (CON) and sodium pyruvate (PYR) $(n=20$ per group). Notably, the GFAP level was robustly reduced relative to groups SUA and SAL, but still significantly higher in group PYR than in group CON. There were statistically significant differences between CON, SUS and SAL from group PYR, respectively ( $\left.{ }^{* *} P<0.01\right)$.

and SAL groups, compared to the CON group, but significant differences were not shown among them. However, it was about doubly increased in the PYR group in comparison to other groups, respectively (Fig. 6). There was statistically significant difference of the NSE expression between groups CON, SUS and SAL from group PYR, respectively ( $\mathrm{P}<0.01)$. Notably, NSE levels were remarkedly higher in the PYR group than in the CON group.

\section{S100B expression}

The relative S100B protein expressions were also significantly declined in the SUS and SAL groups, relative to the CON group, respectively $(\mathrm{P}<0.05)$. It was worth noting that the S100B expression was around 3-4 folds increased in the PYR group in comparison to other groups, respectively (Fig. 7). There was a statistically significant difference of the S100B expression between the CON, SUS and SAL groups from the 

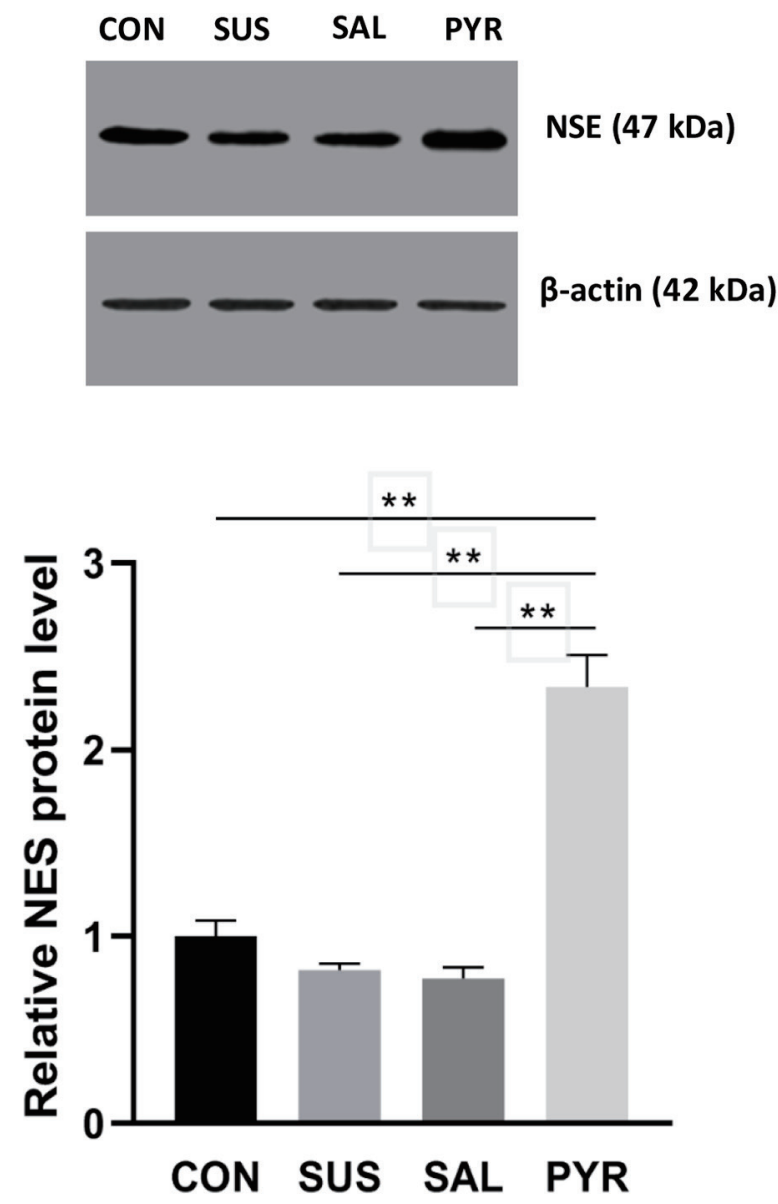

Figure 6. Relative neuron-specific enolase (NES) expressions by Western blotting in L5 dorsal root ganglia (DRG) nerve cells in various treatment groups. The NES protein expression was very significantly enhanced in group sodium pyruvate (PYR) relative to groups control (CON), suspension (SUS) and normal saline (SAL), respectively $\left(n=20\right.$ per group; ${ }^{*} \mathrm{P}<0.01$ ). There were not significant differences among group CON, SUS and SAL though the expressions were lower in groups SUS and SAL relative to group CON, respectively.

PYR group, respectively $(\mathrm{P}<0.01)$. The S100B level of group PYR was also notably higher relative to group CON.

\section{Pyruvate effects on ATP and ATPase in L5 DRG}

\section{ATP content}

ATP contents were decreased in all three suspension groups, relative to the CON group. The ATP content (nmol/gprot) was reduced by $60 \%$ and $61 \%$ in the SUS and SAL groups than in the CON group, respectively. However, ATP levels were 35\% and 38\% higher in group PYR than in groups SUS and SAL, respectively. There was a statistically significant difference of groups SUS and SAL from groups CON and PYR $(\mathrm{P}<0.05 ; \mathrm{P}<$ 0.01; Fig. 8A). But the ATP level was still significantly reduced in the PYR group, compared to the CON group $(\mathrm{P}<0.01)$.
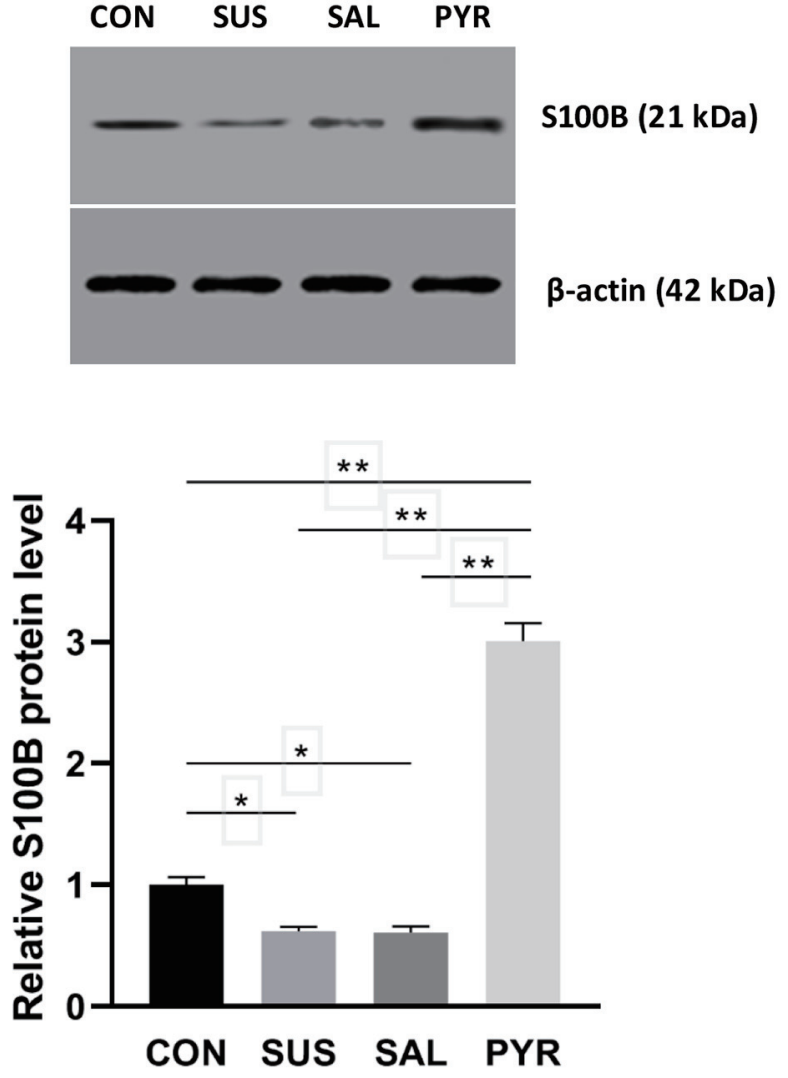

Figure 7. Relative $\mathrm{S} 100$ calcium-binding protein $\mathrm{B}(\mathrm{S} 100 \mathrm{~B})$ expressions by Western blotting in L5 dorsal root ganglia (DRG) nerve cells in various treatment groups. The S100B protein expression was very significantly increased in group sodium pyruvate (PYR) relative to groups control (CON), suspension (SUS) and normal saline (SAL), respectively $(n=20$ per group; $\left.{ }^{* *} \mathrm{P}<0.01\right)$, whereas the expressions in group SUS and SAL were significantly decreased relative to group CON, respectively ( ${ }^{*} \mathrm{P}<$ $0.05)$, but no difference was seen between group SUS and group SAL.

\section{ATPase activity}

ATPase activities were reduced in all three suspension groups in comparison with the CON group. The ATPase activity $(\mu \mathrm{mol} / \mathrm{mgprot} / \mathrm{h})$ in the SUS and SAL groups was reduced by $57 \%$ and $59 \%$ from the CON group, respectively. However, the activities were $42 \%$ and $51 \%$ higher in group PYR than in groups SUS and SAL, respectively. There was a statistically significant difference of groups SUS and SAL from groups CON and PYR ( $<<0.01$; Fig. 8B). Notably, the ATPase activity in the PYR group was still significantly decreased, compared with the CON group $(\mathrm{P}<0.01)$.

The general activity of rats in the experiment was normal except HU, and no tail injury or death was reported.

\section{Discussion}

The female rats were excluded in the experiment due to the sexual difference of DRG damage following HU [5]. 

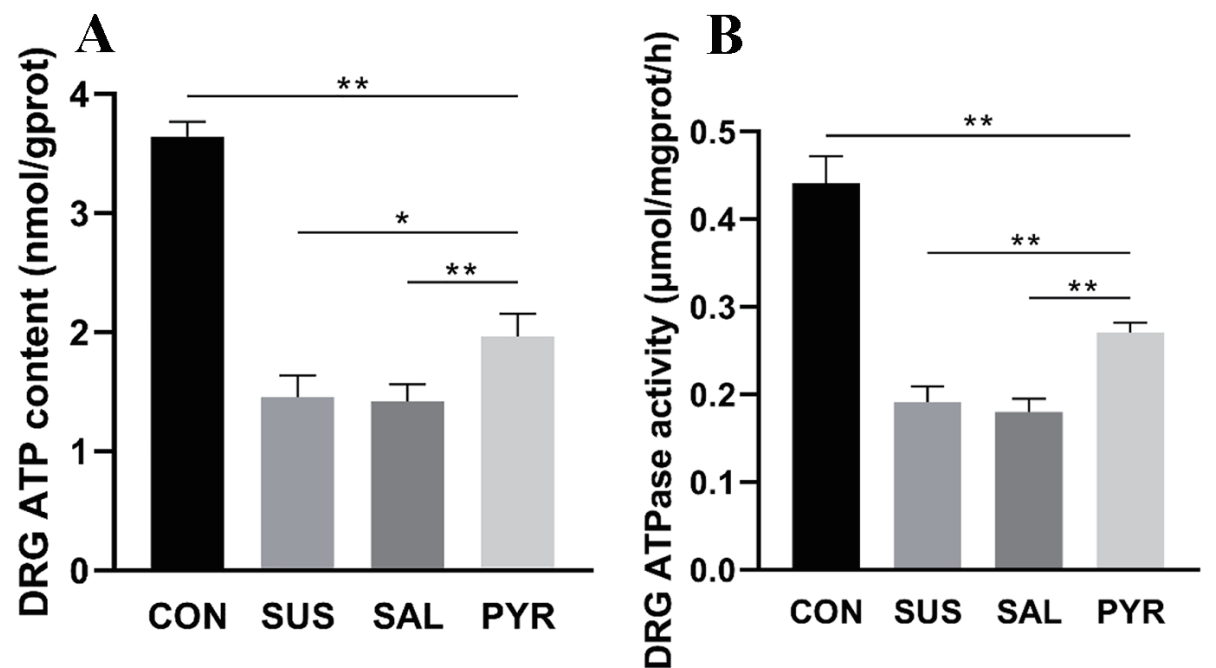

Figure 8. (A) Adenosine triphosphate (ATP) contents in L5 dorsal root ganglia (DRG) tissues in various treatment groups. The ATP contents were decreased in all three suspension groups. There were statistically significant differences between groups suspension (SUS) and normal saline (SAL) from groups control (CON) and sodium pyruvate (PYR) $(n=20$ per group). However, the ATP level was still significantly lower in group PYR compared with group CON. There was a statistically significant difference between groups SUS and SAL from group PYR, respectively ( ${ }^{*} P<0.05$; $\left.{ }^{* *} P<0.01\right)$. (B) Adenosine triphosphatase (ATPase) activities in L5 DRG tissues in various treatment groups. The ATPase activity was reduced in all three suspension groups. There were statistically significant differences between the SUS and SAL groups from the CON and PYR groups $(n=20$ per group). However, the ATPase activity in group PYR was still significantly reduced relative to group CON. There were statistically significant differences between groups CON, SUS and SAL from group PYR, respectively $\left({ }^{* *} P<0.01\right)$.

\section{Major findings of pyruvate protection}

\section{Changes in morphology}

The DRG are most of neurons that convert external pain stimulation into internal nerve impulses and transmit them to the spinal cord. However, pathological pain stimulation is induced due to changes in cellular and myelin structure and function when the DRG suffer from ischemia or innutrition. As demonstrated in the alterations of L5 DRG microstructure and function under $\mathrm{HU}[3,5]$, data here further discovered that the microgravity in rats of groups SUS and SAL resulted in significant morphological alterations in DRG neurons: quite swelling, gap widened, fine granular basophilic materials and cytoplasmic vacuoles appeared as shown in the H\&E staining, whereas cell features were almost undistinguishable in group PYR from those in group CON without experiencing $\mathrm{HU}$ (Fig. 1).

The Nissl bodies are composed of rough endoplasmic reticulum and free polyribosomes in DRG neurons. The functional status of Nissl body can determine the synthesis of nerve growth factors and its morphology can reflect the functional activity of neurons [18]. The altered structure or decreased content of the Nissl body staining indicates function and survival of neurons impaired, which reduces the synthetic proteins and relevant nutritional factors [19]. The results here showed that after HU for 8 weeks, the amount and structure of stained Nissl neurons in groups SUS and SAL were seriously decreased and impaired under a light microscope, while the changes in group PYR were slight and close to those in group CON (Fig. 2A, B). In addition, changes in immunofluorescence of dgen-MBP of
L5 DRG were comparably consistent with alterations above in all the groups experienced the 8-week HU (Fig. 3A, B).

These morphological changes confirmed that HU caused DRG injury in the rats, as previously reported [5]. Also, these preliminary results first showed that in the early stage of DRG lesion induced by microgravity, the microstructure damage could be significantly attenuated by oral pyruvate. Interestingly, ethyl pyruvate (EP, a derivate of sodium pyruvate) was recently reported with an analogical protection of the Nissl staining against motor neurons injury in the spinal cord induced by ischemia reperfusion, but EP was delivered via peritoneal injection, not orally ingested [20].

\section{Changes in cellular function}

The GDNF has a strong neurotrophic effect on neurons in the prevention of their degeneration and can rescue cells from apoptosis as well as their death following injury. As demonstrated that the DRG injury led to GDNF reduction previously [5], present data of Western blotting displayed that relative GDNF protein expressions in the SUS and SAL groups were significantly decreased, relative to those in the CON and PYR groups following HU for 8 weeks. On contrary, the GDNF expression in the PYR group was surprisingly accumulated to over two times of those in the SUS and SAL groups and even more than that in the CON group. The data of GDNF mRNA detection in all the groups were also comparable with alterations above (data not shown) [21]. The present results merely exhibited that the GDNF protein in DRG injury had a prominent feedback increase with chronic pyruvate supplementation in group 
PYR during an 8-week period of microgravity in rats (Fig. 4). Although the pyruvate neuroprotection is well known, at present, the underlying reasons of profoundly increased GDNF protein by pyruvate remain unknown. To date, there is no report concerning the pyruvate stimulation of GDNF expression, in vivo, though EP was also shown with an in parallel effect in primary astrocyte cultures, in vitro [22]. Intriguingly, the histone deacetylase inhibitors (HDACi), like sodium butyrate, up-regulate GDNF gene transcription through the HDAC inhibition in astrocytes to protect dopaminergic neurons [23], while pyruvate is one of HDACis [24]. Nevertheless, present data revealed that oral pyruvate could robustly stimulate the GDNF secretion, which attenuated DRG damage during HU.

The GFAP is a unique protein in astrocytes cytoskeleton of DRG, the main element of intermediate filament network which assures cell integrity and resilience. It is believed to be a marker of the maturation of astrocytes. As a cell signal transduction pathway, GFAP is largely involved in neurons' structure and metabolism [25]. The expression of GFAP is increased in response to injury, neurodegenerative disease and aging, but hardly expressed under physiological status. Data here discovered that relative GFAP expressions were significantly enhanced in the SUS and SAL groups in comparison with those in the CON and PYR groups (Fig. 5). It indicated that astrocytes in DRG injury were activated and the GFAP predominant expression might be closely associated with the DRG damage repair. The GFAP expression was significantly decreased in the PYR group than in both SUS and SAL groups, but still was significantly enhanced than that in the CON group, suggesting that oral pyruvate could be beneficial in neuroprotective effects on DRG tissues damaged in the HU injury, as previously demonstrated that pyruvate could control the reactive astrocyte proliferation (gliosis) and maintain the morphological integrity of astrocytes in various injuries [26, 27]. The detection of GFAP mRNA also showed a similar manner of changes in these groups (data not shown). However, current pyruvate dosage could not fully prevent the damage. Again, EP reduced the GFAP expression from proliferating astrocytes in rats subjected to experimental encephalomyelitis [28].

Interestingly, results here indicated that oral pyruvate promoted the GDNF and diminished the GFAP in DRG nervous tissues during HU. The results suggest that pyruvate like GDNF and GFAP may play an important role in preserving environmental stability, plasticity repair and neuronal survival in nervous systems [25].

Both NES and S100B proteins are clinical sensitive biomarkers in various damages of nerve systems. Both are elevated from extracellular fluids in the early stage of damage but declined afterwards as early clinical predictors of prognosis $[29,30]$. As shown in the patients, NES and S100B expressions were decreased in groups SUS and SAL than in group $\mathrm{CON}$ in an 8-week $\mathrm{UH}$, though there were no statistically significant differences in NES levels among these three groups. However, the levels were first shown to enhance 2-3 folds in group PYR, compared to group CON in both NES and S100B (Figs. 6 and 7). It is well known that exogenous pyruvate is neuroprotective, but the causes of profoundly enhanced NES and S100B levels, as the GDNF expression shown above, remain at present unknown. The in vitro mimic cellular experiments are needed for exploring underlying mechanisms of the pyruvate protection in these respects.

The ATP generation and the ATPase activity are direct biomarkers of cellular energy metabolism. Present results demonstrated that cellular energy metabolism in DRG tissues was evidently inhibited by HU injury and oral pyruvate profoundly preserved energy metabolism in group PYR during the microgravity (Fig. 8A, B), therefore, DRG tissues' structure and function were mostly preserved. Although the ATP level and the ATPase activity in the PYR group were mostly preserved, they were still robustly inferior to those in the CON group. Metabolic studies illustrate that glycolysis is usually promoted with reduction of oxidative phosphorylation in weightlessness [31,32]. Blood pyruvate from the gastrointestinal truck can readily enter mitochondria via mitochondrial pyruvate carrier, reactivate the inhibited pyruvate dehydrogenase $(\mathrm{PDH})$ to near normal context and restore the tricarboxylic acid (TCA) cycle function, as demonstrated in shock and diabetic animals $[33,34]$; pyruvate anti-oxidative stress and anti-inflammation might be also beneficial in the TCA cycle metabolism additionally as an energy substrate. All facilitated the ATP generation and the ATPase activity in the experiment.

Taken all together, the results preliminarily illustrated that the morphological alteration in group PYR was preserved close to those in group $\mathrm{CON}$, while energy metabolic disorder and cellular dysfunction in DRG nervous tissues were markedly illuminated by oral pyruvate, but not fully recovered from the HU injury with the current pyruvate dosage. The differences of histopathological changes from metabolic and functional alterations suggest that the metabolic aberrance induced by HU early occur prior to morphological changes. The doseeffect curve remains to be explored.

\section{Oral pyruvate implication in spaceflight}

Present findings clearly illustrated that oral pyruvate improved the histopathological alteration and metabolic dysfunction of DRG tissues in rats subjected with HU for 8 weeks. The results provide additional evidence that oral $0.35-4 \%$ pyruvate as drinking water did protect from alterations in the structure and function of nervous systems induced with various insults [11, 35-37]. Newly, humans brain parenchyma injury was evidenced following a long-duration spaceflight, as indicated with increased blood biomarkers: GFAP, NfL (neurofilament light chain protein) and amyloid $\beta$ from five cosmonauts [38]. As demonstrated previously, pyruvate also protects against amyloid- $\beta$-induced neuronal cell damage [39]. Accordingly, oral pyruvate may be very beneficial in the attenuation or prevention of DRG damage, probably including SCI and other metabolic disorders and organ dysfunction, for astronauts in spaceflight.

Oral pyruvate $(0.55 \%)$ in this study was selected following our prior experiences. The optimal dosage and the ideal concentration of oral pyruvate fluids for astronauts need to be further explored. It is worth noting that a large dosage of oral and intravenous (IV) pyruvate had shown safety and effectiveness in several preliminary clinical trials with the absence of clinical toxicity [40]. However, oral sodium pyruvate alone in less than $10.0 \mathrm{~g}$ is not effective with no plasma pyruvate elevation in humans, but large dosages are of gastrointestinal irritation symptoms [40]. Alternatively, the novel pyruvate fluid in Pyr- 
ORS $(0.35 \%$ pyruvate and $1.35 \%$ glucose $)$ effectively restored neuronal structure and function in hippocampal CA1 regions of rats with asphyxial cardiac arrest [11]. In addition, 1\% pyruvate and Pyr-ORS as drinking water similarly demonstrated to renovate the pyruvate dehydrogenase $(\mathrm{PDH})$ activity in the kidney and to protect against nephropathy in diabetic $d b / d b$ mice [34].

It is ascertained that opposite to equal sodium pyruvate alone, oral Pyr-ORS robustly elevated pyruvate in blood and neurons in shocked rats [11, 41], as IV pyruvate shown [42]. Thus, ingestion of a small dose of pyruvate with glucose can be easily absorbed from the intestine via $\mathrm{Na}^{+}$-glucose cotransporter located in intestinal mucosa in the mammalian gut $[7,11,34,41]$. Always drink low pyruvate-enriched water while feeding, thus pyruvate is easily absorbed to blood in rats, but not in humans when the drinking water contains pyruvate only without glucose. The pyruvate consumed in this experiment for each rat was close to around $15.0 \mathrm{~g} / \mathrm{day}$, if a 70-kg person was experienced. In a shock resuscitation from rats, the sum of enteral pyruvate in Pyr-ORS equal to $10.5 \mathrm{~g}$ was ingested, extrapolating to humans [7]. All these data from the rodent study, therefore, clearly illustrate that about $10.0 \mathrm{~g}$ of oral pyruvate in a formula as Pyr-ORS as a functional drink daily may be feasible and beneficial for astronauts in space missions.

Finally, albeit EP appears neuroprotective [20, 22, 28], it is distinctly different from pyruvate of sodium salt; the EP clinical trial failed over a decade ago $[40,43]$, leading to its hopeless in a clinical perspective. However, further studies with oral pyruvate are warranted particularly in underling metabolic and molecular pathways of the pyruvate protection of DRG in microgravity conditions.

\section{Limitations}

1) Present data were not to substantiate the precise causal effect of pyruvate protection on the L5 DRG neurons, but aimed to, in vivo, a principal proof of oral pyruvate effects on rats subjected with the HU injury. However, cell experiments, in vitro, to illustrate the intimate effects of pyruvate on DRG neurons following HU would further prompt the hypothesis and facilitate addressing the underlying metabolic and molecular mechanisms of pyruvate actions. 2) Current immunofluorescent expression of MBP was simple. If additional double immunofluorescence with anti-neurofilament antibody was conducted, pictures would be more in a clear and appealing format with the pyruvate treatments. 3) Oral Pyr-ORS (0.35\% and $1.35 \%$ glucose) was not included in the experiments, nor pyruvate dose-effect curve was explored.

\section{Conclusion}

Present data further demonstrate that microgravity induces significant L5 DRG damage in tissue morphology and cell function of rats subjected to HU for 8 weeks. The results first indicate that oral pyruvate in Pyr-ORS may effectively prevent the DRG tissues from HU injury with clinical feasibility. The oral pyruvate formula remains explored for the optimal dosage and efficacy for astronauts' use in space missions.

\section{Acknowledgments}

None to declare.

\section{Financial Disclosure}

The Foundation of State Key Lab of Space Medicine Fundamentals and Application, China (SMFA15K03) provided supports; Dr. Cui $\mathrm{G}$ received the fund. The fund provider was not associated with the experimental design, data analysis, discuss and submission for publication.

\section{Conflict of Interest}

None to declare.

\section{Informed Consent}

Not applicable.

\section{Author Contributions}

Li Y, Cui G and Zhou FQ designed the experiments; Li Y, Ren NT, Chen C, Qi P and Zhang H conducted the animal experiments, collected the data and analyzed results; Zhou FQ and Li Y prepared the manuscript; Cui G and Zhou FQ critically revised the manuscript. All authors read and agreed to submit for publication.

\section{Data Availability}

The data supporting the findings of this study are available from the corresponding author upon reasonable request.

\section{Abbreviations}

ATP: adenosine triphosphate; ATPase: adenosine triphosphatase; dgen-MBP: degenerated myelin basic protein; DRG: dorsal root ganglia; GDNF: glial cell line-derived neurotrophic factor; GFAP: glial fibrillary acidic protein; HU: hindlimb unweighting; NSE: neuron-specific enolase; L5: lumbar 5; S100B: S100 calcium-binding protein B

\section{References}

1. Willey JS, Britten RA, Blaber E, Tahimic CGT, Chancellor J, Mortreux M, Sanford LD, et al. The individual and 
combined effects of spaceflight radiation and microgravity on biologic systems and functional outcomes. J Environ Sci Health C Toxicol Carcinog. 2021;39(2):129-179.

2. Chang DG, Healey RM, Snyder AJ, Sayson JV, Macias BR, Coughlin DG, Bailey JF, et al. Lumbar spine paraspinal muscle and intervertebral disc height changes in astronauts after long-duration spaceflight on the international space station. Spine (Phila Pa 1976). 2016;41(24):19171924.

3. Ren JC, Fan XL, Song XA, Zhao XH, Chen MX, Shi L. Prolonged hindlimb unloading leads to changes in electrophysiological properties of L5 dorsal root ganglion neurons in rats after 14 days. Muscle Nerve. 2012;45(1):6569.

4. Islamov RR, Rizvanov AA, Tyapkina OV, Shenkman BS, Kozlovskaya IB, Nikolskiy EE, Grigoryev AI. Genomic study of gene expression in the mouse lumbar spinal cord under the conditions of simulated microgravity. Dokl Biol Sci. 2011;439:197-200.

5. Zhang H, Ren NT, Zhou FQ, Li J, Lei W, Liu N, Bi L, et al. Effects of hindlimb unweighting on MBP and GDNF expression and morphology in rat dorsal root ganglia neurons. Neurochem Res. 2016;41(9):2433-2442.

6. Flaherty DC, Hoxha B, Sun J, Gurji H, Simecka JW, Mallet RT, Olivencia-Yurvati AH. Pyruvate-fortified fluid resuscitation improves hemodynamic stability while suppressing systemic inflammation and myocardial oxidative stress after hemorrhagic shock. Mil Med. 2010; 175(3):166-172.

7. Yu W, Hu S, Xie ZY, He ZJ, Luo HM, Lin HY, Zhou FQ, et al. Pyruvate oral rehydration solution improved visceral function and survival in shock rats. J Surg Res. 2015;193(1):344-354.

8. Hu S, Dai YL, Gao MJ, Wang XN, Wang HB, Dou YQ, Bai XD, et al. Pyruvate as a novel carrier of hydroxyethyl starch 130/0.4 may protect kidney in rats subjected to severe burns. J Surg Res. 2018;225:166-174.

9. Netzahualcoyotzi C, Tapia R. Degeneration of spinal motor neurons by chronic AMPA-induced excitotoxicity in vivo and protection by energy substrates. Acta Neuropathol Commun. 2015;3:27.

10. Scott GF, Nguyen AQ, Cherry BH, Hollrah RA, Salinas I, Williams AG, Jr., Ryou MG, et al. Featured Article: Pyruvate preserves antiglycation defenses in porcine brain after cardiac arrest. Exp Biol Med (Maywood). 2017; 242(10):1095-1103.

11. Bai WP, Li J, Han RL, Gu Y, Sun XD, Ye J, Zhou FQ, et al. Effects of hypotonic pyruvate oral rehydration solution on brain injury in rats subjected to asphyxial cardiac arrest. Chin J Injury Repair \& Wound Healing. 2017;12(5):326-330 (in Chinese with English abstract).

12. Hu S, Liu WW, Zhao Y, Lin ZL, Luo HM, Bai XD, Sheng ZY, et al. Pyruvate-enriched oral rehydration solution improved intestinal absorption of water and sodium during enteral resuscitation in burns. Burns. 2014;40(4):693701.

13. Liu R, Hu XH, Wang SM, Guo SJ, Li ZY, Bai XD, Zhou $\mathrm{FQ}$, et al. Pyruvate in oral rehydration salt improves hemodynamics, vasopermeability and survival after burns in dogs. Burns. 2016;42(4):797-806.

14. Chandrasekharan P, Koenigsknecht C, Helman J, Lakshminrusimha S. Protection from systemic pyruvate at resuscitation in newborn lambs with asphyxial cardiac arrest. Physiol Rep. 2020;8:e14472.

15. Bando $Y$, Nomura $T$, Bochimoto $H$, Murakami K, Tanaka T, Watanabe T, Yoshida S. Abnormal morphology of myelin and axon pathology in murine models of multiple sclerosis. Neurochem Int. 2015;81:16-27.

16. Han Q, Xiang J, Zhang Y, Qiao H, Shen Y, Zhang C. Enhanced neuroprotection and improved motor function in traumatized rat spinal cords by rAAV2-mediated glialderived neurotrophic factor combined with early rehabilitation training. Chin Med J (Engl). 2014;127(24):42204225.

17. Weicker H, Hageloch W, Luo J, Muller D, Werle E, Sehling KM. Purine nucleotides and AMP deamination during maximal and endurance swimming exercise in heart and skeletal muscle of rats. Int J Sports Med. 1990;11(Suppl 2):S68-77.

18. Johnson IP, Sears TA. Target-dependence of sensory neurons: an ultrastructural comparison of axotomised dorsal root ganglion neurons with allowed or denied reinnervation of peripheral targets. Neuroscience. 2013;228:163178.

19. Luo L, Li C, Deng Y, Wang Y, Meng P, Wang Q. Highintensity interval training on neuroplasticity, balance between brain-derived neurotrophic factor and precursor brain-derived neurotrophic factor in poststroke depression rats. J Stroke Cerebrovasc Dis. 2019;28(3):672-682.

20. Nguyen BN, Albadawi H, Oklu R, Crawford RS, Fink MP, Cambria RP, Watkins MT. Ethyl pyruvate modulates delayed paralysis following thoracic aortic ischemia reperfusion in mice. J Vasc Surg. 2016;64(5):1433-1443.

21. Tsybko AS, Ilchibaeva TV, Kulikov AV, Kulikova EA, Krasnov IB, Sychev VN, Shenkman BS, et al. Effect of microgravity on glial cell line-derived neurotrophic factor and cerebral dopamine neurotrophic factor gene expression in the mouse brain. J Neurosci Res. 2015;93(9):13991404.

22. Shin JH, Kim SW, Jin Y, Kim ID, Lee JK. Ethyl pyruvatemediated Nrf2 activation and hemeoxygenase 1 induction in astrocytes confer protective effects via autocrine and paracrine mechanisms. Neurochem Int. 2012;61(1):89-99.

23. Wu X, Chen PS, Dallas S, Wilson B, Block ML, Wang $\mathrm{CC}$, Kinyamu H, et al. Histone deacetylase inhibitors upregulate astrocyte GDNF and BDNF gene transcription and protect dopaminergic neurons. Int J Neuropsychopharmacol. 2008;11(8):1123-1134.

24. Spiegel S, Milstien S, Grant S. Endogenous modulators and pharmacological inhibitors of histone deacetylases in cancer therapy. Oncogene. 2012;31(5):537-551.

25. Middeldorp J, Hol EM. GFAP in health and disease. Prog Neurobiol. 2011;93(3):421-443.

26. Rouleau C, Rakotoarivelo C, Petite D, Lambert K, Fabre C, Bonardet A, Mercier J, et al. Pyruvate modifies glycolytic and oxidative metabolism of rat embryonic spinal cord astrocyte cell lines and prevents their spontaneous transformation. J Neurochem. 2007;100(6):1589-1598. 
27. Sharma P, Karian J, Sharma S, Liu S, Mongan PD. Pyruvate ameliorates post ischemic injury of rat astrocytes and protects them against PARP mediated cell death. Brain Res. 2003;992(1):104-113.

28. Djedovic N, Stanisavljevic S, Jevtic B, Momcilovic M, Lavrnja I, Miljkovic D. Anti-encephalitogenic effects of ethyl pyruvate are reflected in the central nervous system and the gut. Biomed Pharmacother. 2017;96:78-85.

29. Stefanovic B, Duric O, Stankovic S, Mijatovic S, Doklestic K, Stefanovic B, Jovanovic B, et al. Elevated serum protein $\mathrm{S} 100 \mathrm{~B}$ and neuron specific enolase values as predictors of early neurological outcome after traumatic brain injury. J Med Biochem. 2017;36(4):314-321.

30. Park DW, Park SH, Hwang SK. Serial measurement of S100B and NSE in pediatric traumatic brain injury. Childs Nerv Syst. 2019;35(2):343-348.

31. Marsh DR, Campbell CB, Spriet LL. Effect of hindlimb unweighting on anaerobic metabolism in rat skeletal muscle. J Appl Physiol (1985). 1992;72(4):1304-1310.

32. Michaletti A, Gioia M, Tarantino U, Zolla L. Effects of microgravity on osteoblast mitochondria: a proteomic and metabolomics profile. Sci Rep. 2017;7(1):15376.

33. Sharma P, Walsh KT, Kerr-Knott KA, Karaian JE, Mongan PD. Pyruvate modulates hepatic mitochondrial functions and reduces apoptosis indicators during hemorrhagic shock in rats. Anesthesiology. 2005;103(1):65-73.

34. Zhang XM, Deng $\mathrm{H}$, Tong JD, Wang $\mathrm{YZ}$, Ning $\mathrm{XC}$, Yang XH, Zhou FQ, et al. Pyruvate-enriched oral rehydration solution improves glucometabolic disorders in the kidneys of diabetic $\mathrm{db} / \mathrm{db}$ mice. J Diabetes Res. 2020;2020:2817972.

35. Sahenk Z, Yalvac ME, Amornvit J, Arnold WD, Chen L, Shontz KM, Lewis S. Efficacy of exogenous pyruvate in Trembler(J) mouse model of Charcot-Marie-Tooth neuropathy. Brain Behav. 2018;8(10):e01118.
36. Koivisto H, Leinonen H, Puurula M, Hafez HS, Barrera GA, Stridh MH, Waagepetersen HS, et al. Chronic pyruvate supplementation increases exploratory activity and brain energy reserves in young and middle-aged mice. Front Aging Neurosci. 2016;8:41.

37. Zilberter M, Ivanov A, Ziyatdinova S, Mukhtarov M, Malkov A, Alpar A, Tortoriello G, et al. Dietary energy substrates reverse early neuronal hyperactivity in a mouse model of Alzheimer's disease. J Neurochem. 2013; 125(1):157-171.

38. Zu Eulenburg P, Buchheim JI, Ashton NJ, Vassilieva $\mathrm{G}$, Blennow K, Zetterberg H, Chouker A. Changes in blood biomarkers of brain injury and degeneration following long-duration spaceflight. JAMA Neurol. 2021; 78(12):1525-1527.

39. Wang X, Hu X, Yang Y, Takata T, Sakurai T. Systemic pyruvate administration markedly reduces neuronal death and cognitive impairment in a rat model of Alzheimer's disease. Exp Neurol. 2015;271:145-154.

40. Zhou FQ. Pyruvate-enriched fluids as a novel medical solution and beverage. Food Sci \& Nutri Tech. 2021;6(1):1-7.

41. Liu R, Wang SM, Li ZY, Yu W, Zhang HP, Zhou FQ. Pyruvate in reduced osmolarity oral rehydration salt corrected lactic acidosis in sever scald rats. J Surg Res. 2018;226:173-180.

42. Hu S, Bai XD, Liu XQ, Wang HB, Zhong YX, Fang T, Zhou FQ. Pyruvate Ringer's solution corrects lactic acidosis and prolongs survival during hemorrhagic shock in rats. J Emerg Med. 2013;45(6):885-893.

43. Bennett-Guerrero E, Swaminathan M, Grigore AM, Roach GW, Aberle LG, Johnston JM, Fink MP. A phase II multicenter double-blind placebo-controlled study of ethyl pyruvate in high-risk patients undergoing cardiac surgery with cardiopulmonary bypass. J Cardiothorac Vasc Anesth. 2009;23(3):324-329. 